\title{
Editorial for the 25th anniversary issue
}

\author{
Dieter Jungnickel $^{1} \cdot$ Jennifer Key $^{2} \cdot$ Chris Mitchell $^{3}$. \\ Ronald Mullin $^{4,6}$. Peter Wild ${ }^{5}$
}

Published online: 21 November 2015

C) Springer Science+Business Media New York 2015

The first issue of Designs, Codes and Cryptography (DCC) appeared 25 years ago, in May 1991. Since then, the journal has seen remarkable growth, both in size and in reputation, and it seemed appropriate to mark the journal's silver jubilee with a special volume-an idea first suggested by our publisher, Melissa Fearon, about a year ago. The result is the present volume which has been edited by five of the six former and current Editors-in-Chief; sadly, one of the founding chief editors of the journal, our friend and colleague Scott Vanstone, passed away in March 2014.

DCC rarely publishes survey articles, and then by invitation only. For this special occasion, however, it seemed best to us to have a collection of surveys covering some active and important areas illustrating the journal's scope, and written by leading experts.

It also seems appropriate to review the journal's history in this editorial. During one of Jungnickel's visits to the University of Waterloo (actually, over a pleasant lunch at the Faculty Club), two of the three founding Editors-in-Chief-Dieter Jungnickel and Scott Vanstoneconceived the idea of starting a new high-level journal, which led to the foundation of DCC in 1990, jointly with Ron Mullin. The intention behind this is best seen in the following quote from the editorial for the inaugural issue (Volume 1:1, May 1991):

This journal Designs, Codes and Cryptography (DCC) was founded to meet the needs of mathematicians, engineers and computer scientists working in the areas of design theory, coding theory and cryptography, whose interests extend beyond the bounds of any one of the individual disciplines. We believe that the journal will appeal to

$凶$ Peter Wild

peterrwild@gmail.com

1 Institut für Mathematik, University of Augsburg, 86135 Augsburg, Germany

2 Department of Mathematical Sciences, Clemson University, Clemson, SC 29634, USA

3 Department of Math, Royal Holloway, University of London, Egham, Surrey TW20 0EX, UK

4 Florida Atlantic University, Boca Raton, FL, USA

5 University of London, London, UK

6 University of Waterloo, Waterloo, ON, Canada 
those mathematicians with an interest in seeing deep theoretical results in discrete mathematics applied, to those engineers who appreciate the beauty and usefulness of discrete mathematics and to those computer scientists who are interested in data security, complexity issues and reliable data transmission and storage.

The areas of designs and codes are relatively new disciplines which have grown rapidly in the last three or four decades and continue to evolve. Although cryptography has a much longer history, only recently has it been placed in a more theoretical framework and attracted wide interest from researchers in computer science, engineering and mathematics. There are currently a number of journals which deal with each of the individual disciplines covered in DCC but none which encourages the interaction of the three. Many researchers have found themselves in the situation where they have very interesting results crossing several areas yet falling outside the bounds of most traditional journals in design theory, coding theory or cryptography. One of the aims of this journal is to provide a forum for high quality papers of both a theoretical and a practical nature which bridge more than one of these disciplines. To restrict the journal to only such papers would be a mistake since the applicability of some results to other areas is not always immediately evident. For this reason the framework in which we want to establish DCC is wider. It is not desirable to define the scope of the journal too narrowly but if we have to give such a definition in a single sentence it would be: Papers emphasizing the algebraic and geometric aspects of any of the areas (designs, codes or cryptography) will he within the purview with papers touching more than one of the areas being especially welcome.

These ideas met with considerable enthusiasm when Scott approached Rob Holland at Kluwer Academic Publishers, where he had published his book An Introduction to Error Correction Codes with Applications (jointly with Paul van Oorschot) a few years earlier, and led very quickly to the actual foundation of DCC. (The journal became a Springer journal in 2005.) DCC started with one volume of four issues per year, but soon expanded to two volumes with three issues each in 1995, then to three volumes with three issues each in 1997 , and finally to the current four volumes with three issues each since 2005. While this format has not changed, the steadily increasing number of submissions resulted on the one hand in publishing more papers per issue than before and on the other hand in progressively stricter and more selective standards of reviewing submissions. While this sometimes brings about hard choices for both editors and contributors, it has also increased the quality (and the prestige) of the journal—not an unpleasant development.

The aims and scope of the journal are much the same as at the time of its founding, though they have, of course, evolved somewhat. In addition to the three areas in its name, DCC also publishes a substantial number of articles about finite fields and finite geometries. Finite fields are an indispensable tool for both the construction and the actual application of designs, codes and cryptographic systems, and therefore we include (mostly theoretical) papers on computational aspects of these algebraic structures. Finite geometry has had interesting applications in our areas for a long time, but its role in these areas has been growing considerably over recent years, in particular, in coding theory (as witnessed by hot topics such as LDPC codes and network coding). For the reasons given in the above quote, we also include a certain number of high quality articles in finite geometry that seem of merely theoretical interest at present. Finally, there is a substantial number of articles in sequence design, as sequences with interesting correlation properties come up in all of our areas, and frequently even admit equivalent formulations in these areas. 
The remarkable continuity of the journal is also reflected in the fact that DCC had only six Editors-in-Chief over its first 25 years, with always three serving at any given time. Two of the founding editors retired comparatively early on: Ron Mullin in 1997, and Scott Vanstone in 1999; their duties were taken over by Jennifer Key and Peter Wild, respectively. Peter in turn retired at the end of 2010, and was succeeded by Chris Mitchell. Fortunately, the former Editors-in-Chief keep an interest in the journal's well-being and continue to be involved as honorary editors (as exemplified by this volume), albeit no longer doing the day-to-day chores involved in editing.

It remains to express our sincere thanks to the members of our editorial board for their hard work and to the editorial staff at Springer for their continuous support, in particular, to our publisher Melissa Fearon. Thanks are also due to our readership, to all contributors to the journal, and to the many colleagues who serve as reviewers. Without their dedication, DCC simply could not exist.

Finally, we specifically wish to acknowledge the authors of this special volume, who agreed to write their surveys at rather short notice and who even kept to the very tight deadlines. We are confident that the reader will find this volume interesting and useful, and we hope that the surveys in it will lead to many further exciting developments in our very active areas. We look forward to this, and we also hope and believe that the success of this journal will continue accordingly. 